\title{
Poor diet quality and adverse eating behaviours in young survivors of childhood cancer
}

\author{
Catharine Fleming ${ }^{1}$, Alexia Murphy-Alford ${ }^{2}$, Jennifer Cohen ${ }^{3}$, Mike Fleming $^{4}$, Claire \\ Wakefield $^{5}$, and Fiona Naumann ${ }^{6}$ \\ ${ }^{1}$ Western Sydney University - Campbelltown Campus \\ ${ }^{2}$ University of Queensland \\ ${ }^{3}$ University of New South Wales Faculty of Medicine \\ ${ }^{4}$ Science Division \\ ${ }^{5}$ University of NSW \\ ${ }^{6} \mathrm{QUT}$
}

January 19, 2021

\begin{abstract}
Background The long-term impact of childhood cancer treatment on dietary intake is likely to be complex and the length of time dietary behaviours are affected after childhood cancer treatment is unknown. Aim The aim of this study was to determine the diet quality in childhood cancer survivors recently off treatment and identify possible contributing factors that may affect diet quality in this population. Methods Participants were 65 parents and/or carers of childhood cancer survivors (CCS) (aged 2-18 years), recently off treatment and 81 age-matched controls. Methods Participants completed two self-administered dietary intake and eating behaviour questionnaires. Study data was explored to determine between group differences, bivariate analysis using Spearman's correlations was used to determine the relationship between diet quality and identified variables, and hierarchical cluster analysis was completed to characterise specific variables into clusters. Results CCS had a significantly poorer diet quality score than the age-matched controls $(\mathrm{t}=-2.226, \mathrm{p}=0.028)$. Childhood cancer survivors had significantly higher parent-reported rates of 'picky eating' behaviour than the control group $(\mathrm{t}=0.106 \mathrm{p}=0.044)$. Factors such as picky eating, emotional overeating and Body Mass Index z-score appeared to drive diet quality in survivors. Conclusions A CCS with picky eating behaviours could avoid complete food groups, have strong food preferences/aversions and over- consume high energy foods to maintain their energy intake, possibly affecting diet quality. The outcomes highlighted the need for a tailored intervention aimed at improving healthy eating behaviours in CCS after treatment for cancer.
\end{abstract}

Title: Poor diet quality and adverse eating behaviours in young survivors of childhood cancer

Authors: Catharine A.K. Fleming ${ }^{*}$, Alexia J. Murphy-Alford ${ }^{2}$, Jennifer Cohen ${ }^{4,5}$, Michael R. Fleming ${ }^{3}$, Claire E. Wakefield ${ }^{4,5}$, Fiona Naumann ${ }^{6}$

1. School of Medical Science, UNSW Medicine, University of NSW Australia.

2. Children's Nutrition Research Centre, Child Health Research Centre, School of Medicine, The University of Queensland, Brisbane, Australia

3. Science Division, Office of Environment and Heritage, Hurstville, NSW, Australia

4. Kids Cancer Centre, Sydney Children's Hospital, High Street Randwick, NSW, Australia

5. School of Women's and Children's Health, UNSW Medicine, UNSW, NSW, Australia

6. Faculty of Health, Queensland University of Technology, Brisbane, Queensland, Australia

\section{Corresponding Author*}


Catharine A.K Fleming

School of Health Sciences, Western Sydney University

Tel:+63 439459155

EMAIL: Catharine.Fleming@westernsydney.edu.au

Word Count : Abstract 238, Text 3,165

Tables: 6

Supporting Information: Table 1

Running Title: Diet quality after childhood cancer treatment

Key words: Diet quality, diet intake, feeding behaviour, childhood cancer, treatment completion

\title{
Abbreviations table
}

\begin{tabular}{ll}
\hline ACAES & Australian Child and Adolescent Eating Survey \\
\hline ACARFS & Australian Child and Adolescent Recommended Food Score \\
ADG & Australian Dietary Guidelines \\
ALL & Acute Lymphatic Leukaemia \\
AML & Acute Myeloid Leukaemia \\
BMI z & Body Mass Index z-score \\
BMI & Body Mass Index \\
CCS & Childhood Cancer Survivor \\
CEBQ & Children's Eating Behaviour Questionnaire \\
CFQ & Child Feeding Questionnaire \\
CR & Cranial Radiation \\
CRT & Cranial Radiation Therapy \\
CVD & Cardiovascular Disease \\
EN & Enteral Nutrition \\
FFQ & Food Frequency Questionnaire \\
KCC & Kids Cancer Centre \\
MetS & Metabolic Syndrome \\
NGT & Nasogastric feeding Tube \\
TPN & Total Parenteral Nutrition \\
RDA & Recommended Daily Allowance \\
RDI & Recommended Daily Intake \\
SCH & Sydney Children's Hospital \\
SEIFA & Socio Economic Index For Area \\
\hline
\end{tabular}

\begin{abstract}
Background The long-term impact of childhood cancer treatment on dietary intake is likely to be complex and the length of time dietary behaviours are affected after childhood cancer treatment is unknown. Aim The aim of this study was to determine the diet quality in childhood cancer survivors recently off treatment and identify possible contributing factors that may affect diet quality in this population. MethodsParticipants were 65 parents and/or carers of childhood cancer survivors (CCS) (aged 2-18 years), recently off treatment and 81 age-matched controls. Methods Participants completed two self-administered dietary intake and eating behaviour questionnaires. Study data was explored to determine between group differences, bivariate analysis using Spearman's correlations was used to determine the relationship between diet quality and identified variables, and hierarchical cluster analysis was completed to characterise specific variables into
\end{abstract}


clusters.Results CCS had a significantly poorer diet quality score than the age-matched controls $(\mathrm{t}=-$ $2.226, \mathrm{p}=0.028$ ). Childhood cancer survivors had significantly higher parent-reported rates of 'picky eating' behaviour than the control group $(\mathrm{t}=0.106 \mathrm{p}=0.044)$. Factors such as picky eating, emotional overeating and Body Mass Index z-score appeared to drive diet quality in survivors. Conclusions A CCS with picky eating behaviours could avoid complete food groups, have strong food preferences/aversions and over- consume high energy foods to maintain their energy intake, possibly affecting diet quality. The outcomes highlighted the need for a tailored intervention aimed at improving healthy eating behaviours in CCS after treatment for cancer.

\section{Background}

Many children with cancer are diagnosed in the first 10 years of life ${ }^{(1)}$. This developmental period includes several key phases of eating and cognitive development which contribute to food preferences, diet intake, energy intake and eating patterns as children grow older ${ }^{(2)}$. The development of food preferences occurs through the interaction of foods, sensory cues (olfactory, tactile and visual sensory systems) and the consequence of ingestion, which may elicit a positive or negative response to the food ${ }^{(2)}$.

Eating is an essential human activity, yet evidence from the general paediatric population suggests that up to $40 \%$ of children aged 3-6 years old experience mealtime difficulties ${ }^{(3)}$ and $25 \%$ have feeding disorders, which can affect a child's diet quality ${ }^{(4)}$. Feeding difficulties in young children can range from chronic feeding disorders to more common behaviours such as food refusal, food aversion, excessive mealtime length, inappropriate behaviours and picky eating ${ }^{(4)}$. A child with picky eating behaviours can avoid complete food groups, have strong food preferences/aversions, over-consume high energy foods to maintain energy intake and demonstrate an unwillingness to try new foods ${ }^{(3)}$.

As in the general paediatric population, children receiving cancer treatment can also have feeding difficulties and poor diet quality during treatment ${ }^{(5)}$, with the consequences having the potential to be magnified in this population. Weight loss during treatment is associated with poorer clinical outcomes such as impaired immune competence, increased susceptibility to infection, reduced treatment tolerance leading to dose reductions and treatment delays, increased side-effects, decreased wound healing and reduced quality of life (6-9). Despite the impact of cancer treatment toxicities, which can result in the child feeling nauseated and unwell, parents often pressure their child to eat during this stressful time to maintain weight during childhood cancer treatment ${ }^{(10)}$. Parent pressure, coupled with food becoming associated with treatment side effects of vomiting/nausea and taste/smell changes can lead to learned food aversions during treatment, restricting adequate intake and compromising diet quality ${ }^{(10,11)}$. During cancer treatment, young children are unable to differentiate between the impact of the disease, treatment and side effects on their eating, placing patients at a high risk of ongoing adverse eating behaviours ${ }^{(12)}$.

With significant therapeutic success and a resulting increase in survival outcomes, the medical challenge has become the minimisation of the secondary effects of cancer treatments ${ }^{(13)}$. One of the most significant findings of childhood cancer survivors (CCS) is the increased risk of developing metabolic syndrome (MetS) and cardiovascular disease $(\mathrm{CVD})^{(14)}$. Poor dietary intake is associated with the development and progression of MetS in the general population as well as adult survivors of childhood cancer ${ }^{(14)}$. What is not known are the long-term implications that interrupted feeding development and changes in eating behaviour during cancer treatment has on dietary intake in childhood cancer survivors and the longer-term impact on health including MetS.

When looking specifically at macro- and micronutrient intake, Cohen et al (2015) found that children who had recently completed cancer treatment had an inadequate micro-nutrient intake and were consuming an excessive energy intake ${ }^{(15)}$. An increase in the intake of high fat, high sugar and refined convenience foods has also been found for cancer survivors ${ }^{(15-17)}$. The impact of childhood cancer treatment on dietary intake in young childhood cancer survivors of is likely to be complex. The combination of treatment toxicities (nausea, food aversions and taste and smell changes) with maladaptive parent eating behaviours all potentially influence dietary intake during and after cancer treatment. While recent research shows that adult 
survivors of childhood cancer are displaying frequent cravings for junk food (18) little is known about the eating behaviours of young survivors of childhood cancer. It is also unknown how the diet quality of young childhood cancer survivors compares with the diet of the general paediatric population.

The primary aim of this study was to compare diet quality of childhood cancer survivors with age-matched children from the general community. Secondly, the study aimed to identify possible contributing factors that may affect diet quality in this population.

\section{Methods}

\section{Participants}

Participants were parents and/or carers of CCS aged between 2-18 years, who had received anti-cancer treatment at Kids Cancer Centre (KCC), Sydney Children's Hospital (SCH), and John Hunter Children's Hospital (JHCH), Newcastle, Australia. Parents and/or carers were eligible if they were the mother, father or primary caregiver of a child who: 1) had undergone treatment for any type of childhood cancer; 2) had completed their cancer treatment protocol; 3) were less than five years' post-diagnosis; and 4) had no cognitive or other mental impairments. Control group participants were parents and/or carers of a child aged between 2-18 years with no co-morbidities that affected nutritional intake. Participants were recruited between September 2012 and March 2014. The study protocol had full ethical approval from SCHN HREC (12/SCHN/29) and UNSW HREC (HC12659).

\section{Recruitment}

Study information packages were posted to eligible participants. To recruit parents of the control group, staff of participating childcare centres, community groups and volunteer community members were contacted to identify eligible participants and to ensure that: a) it was appropriate to approach each family identified; and b) they met the study eligibility criteria. Potential participants were then provided with a study package. Parents returned their completed questionnaires and a signed consent form in the supplied replypaid envelope.

\section{Data collection}

Parents/carers were asked to complete a separate self-report questionnaire for their child's demographic, treatment (if applicable) and eating behaviour information followed by a dietary intake questionnaire. The first questionnaire included information about the child's geographic location (i.e. postcode of residence) and demographic data (i.e. gender, date of birth, weight and height, and current medications). Clinical information included cancer diagnosis, date of diagnosis, date of completion of cancer treatment, cancer treatment regimen (surgery, radiation, chemotherapy and hematopoietic stem cell transplant) and nutrition interventions (education, oral nutrition support (ONS), Enteral Nutrition (EN), Total Parenteral Nutrition (TPN) received during cancer treatment. Parent demographic information collected included: socioeconomic status, gender, date of birth, employment status, education and perceived health status, weight and height.

The child's eating behaviour was measured using the validated parent report Children's Eating Behaviour Questionnaire (CEBQ), which assesses variation in eating style between children. The measure assesses the child's eating style though 35 items, comprising eight subscales(19). An objective measure of the child's food pickiness was determined using the Child Feeding Questionnaire (CFQ), 'picky eating' subscale. The CFQ is a validated seven factor parent self-report measure designed to assess parent attitudes, beliefs and practices regarding their child's eating behaviour ${ }^{(20)}$.

The second questionnaire was the Australian Child and Adolescent Eating Survey (ACAES) which assessed the child's dietary intake. The ACAES is a validated parent reported 120 question self-administered semi-quantitative Food Frequency Questionnaire (FFQ) that requests specific information about foods and beverages consumed by the child to assess their dietary intake ${ }^{(21)}$.

\section{Analysis}


Child and parent weight and height were used to calculate body mass index (BMI), using the formula: weight in kilograms divided by height in metres squared. For each child aged $>2$ years, a BMI z-score was calculated using Epi Info (Version 3.5.1, 2008; Centres for Disease Control and Prevention, USA). This allowed for equal comparison across all age groups ${ }^{(22)}$. Weight and height were also used to determine the weight range category of the child (thinness, healthy, overweight and obese) to define the child's nutritional status $^{(37)}$. The dietary intake, proportion of energy intake and serve size data were calculated using the $\operatorname{ACAES}^{(21)}$.

The Australian Child and Adolescent Recommended Food Score (ACARFS) was generated from the ACAES to determine each child's diet quality. This score reflected the relationship between the child's dietary diversity and nutrient adequacy by measuring their adherence to the Australian dietary guidelines ${ }^{(24)}$.

Study data were explored using univariate data analysis from the statistical software package SPSS (version 22, 2014; SPSS Inc, Chicago, IL, USA) to assess whether there were significant differences between the survivor and control groups. Chi-squared and t-tests (two-tailed, $\alpha=0.05$ ) were used to compare parent/child demographics, BMI z-scores and Socio-Economic Indexes for Areas (SEIFA) index. Age and sex adjusted means of the ACARFS were compared using analysis of covariance to determine the difference between age and gender for diet quality scores. Differences between the parent/child eating behaviours sub-scales determined in the CEBQ and CFQ were compared using t-tests.

Bivariate analysis using Spearman's correlations was used to determine the relative contribution of child treatment regimen side effects, parent feeding behaviour and child eating behaviour on the child's diet quality. Differences and associations were considered significant at $\mathrm{p}<.05$ (2-tailed) for all tests.

For the quantitative data variables, hierarchical cluster analysis was completed using the software ' $R$ ' (version 3.0.1; Packages: 'mclust' version 4.3; 'FactoMineR' version 1.27). This analysis grouped participants characterised by specific variables into clusters based on the distance between each observation or variable. In this study, four composite scores derived from the questionnaires (BMI z score, child picky eating behaviour, child emotional overeating behaviour and diet quality) were used after scaling and a logit transformation.

\section{Results}

\section{Demographics}

A total of 146 parents of children aged 2-18 years completed the study: 65 participants were parents of CCS (response rate 26\%) and the control group of 81 participants were parents of children without a cancer diagnosis (response rate $64 \%$ ).

Significant differences between control and cancer groups were for parent age, education level, and employment, with control parents working more hours per week. There was no significant difference in BMI z-scores for participating children, although the CCS had a higher proportion of participants classified as being overweight ( $21 \%$ vs $6 \%$ ) and having grade 1 thinness (12\% vs $2 \%$ ) (TABLE 1 ).

In the CCS group, mean age at diagnosis was $6.2( \pm 4.5)$ years, mean time since diagnosis was $50.3( \pm 15.6)$ months and mean time since treatment completion was $24.9( \pm 17.3)$ months. CCS had a heterogeneous representation of cancer diagnoses, with the most common being Acute Lymphoblastic Leukaemia (ALL) $29 \%(\mathrm{n}=19)$ (TABLE 2).

\section{Nutritional intake}

There was no significant difference in overall daily energy intake between CCS and the control group ( $\mathrm{t}=1.738$, $\mathrm{p}=0.84$ ). Children with cancer consumed significantly more sweetened beverages on a daily basis than the control group ( $\mathrm{z}=-1.978, \mathrm{p}$ 0.048). There was no statistically significant difference between CCS and the control group for all other core or non-core food intake (TABLE 3).

Diet quality 
The CCS had poorer diet quality than children in the control group $(\mathrm{t}=-2.226, \mathrm{p}=0.028)$. There was no significant difference between the number of CCS and controls categorised into poor, moderate and good diet quality groups, however $54 \%$ of CCS were considered to have a moderate to good diet quality compared with $71 \%$ of healthy control participants (TABLE 4).

\section{Child feeding behaviour}

Using the CEBQ, CCS were found to have a higher percentage (40\%) of participants classified as having 'food fussiness' than healthy controls $(28 \%)$. A significant difference $(\mathrm{t}=0.106 \mathrm{p}=0.044)$ was found between CCS and the control group when objectively measuring picky eating behaviour, using the CFQ (picky eating subscale) ${ }^{(19)}$

\section{(TABLE 5).}

For the CCS group there was an inverse relationship between the picky eating score and age at diagnosis ( $\mathrm{Rho}=-0.314, \mathrm{p}=0.035)$ and current age $(\mathrm{Rho}=-0.323, \mathrm{p}=0.031)$, with CCS diagnosed at a younger age having higher picky eating scores. An inverse relationship was also found between children's emotional overeating score and the type of cancer treatment regimen they received (Rho=-0.333, $\mathrm{p}=0.025$ ), with CCS that had less intensive treatment (such as surgery or only one treatment modality of chemotherapy OR radiation) being more likely to have parent-reported overeating behaviours.

\section{Analysis of factors contributing to diet quality outcomes}

Of the 146 participants, 114 had a BMI z-score and were included in the cluster analysis. Of these, 55 were CCS and 59 were age-matched controls. The cluster analysis used four factors: child BMI z-score, ACARFS score, child emotional overeating score and child picky eating score, and the generated hierarchy was accepted at the three-cluster level. The allocation of healthy controls and CCS to these three groups is shown in Table 6 , with all three clusters being a mixture of participants.

The first cluster (normal eating behaviour) was characterised by children with a healthy BMI z-score, good diet quality and low altered eating behaviours (picky eating/emotional overeating) and included 15 (27\%) CCS and 29 (49\%) controls. The second cluster (low BMI z-score, altered eating behaviour and poor diet quality) had the smallest number of participants with 8 (15\%) CCS and 4 (8\%) control. This cluster was characterised by children with a low (underweight) BMI z-score, poor diet quality and altered eating behaviour, particularly picky eating. Cluster 3 (high BMI z-score, altered eating behaviour and poor diet quality) was characterised by children with a high BMI z-score (trending to overweight), poor diet quality and altered eating behaviour, particularly overeating (32 (58\%) CCS and 26 (44\%) controls (TABLE 6).

Further analysis of the survivor group was undertaken to describe the treatment received by each child in each group. Children who had Non-Hodgkin's lymphoma and Wilms' tumour were found to have the highest proportion of CCS in cluster 3, with brain tumour survivors having the highest proportion of children in cluster 2 (Supplementary Table 1).

\section{Discussion}

This study revealed that CCSs consume diets of poorer quality and have higher rates of picky eating than their age-matched peers in the control group. It also appears that CCS eating behaviour and diet quality may be influenced by treatment intensity and the child's cancer diagnosis.

Both the CCS group and the control group were found to have poor dietary intake for vegetables, grains, meat protein and dairy, affecting overall diet quality. These findings are in line with global patterns of dietary intake in CCSs. Zhang et al (2015) reported young childhood cancer survivors had poor adherence to the US dietary guidelines and consequently poor diet quality. This poor diet quality of CCS is of concern, as CCS are at a higher risk for early mortality and CVD than their peers ${ }^{(16)}$. There is also a known association between poor dietary intake and many chronic diseases such as cardiovascular disease, metabolic syndrome and type 2 diabetes in the general population ${ }^{(25)}$. 
This evidence of poor adherence to dietary guidelines in CCS needs to be addressed in young cancer survivors. The finding of low vegetable intake for childhood cancer survivors is of particular concern due to the importance of folic acid intake and homocysteine levels ${ }^{(17,26)}$. Decreased folic acid intake through a reduced intake of green leafy vegetables can result in an increase in homocysteine levels which are associated with endothelial dysfunction, increased low-density lipoprotein levels and ultimately atherosclerosis which can lead to an increased risk of $\mathrm{CVD}^{(17,26)}$.

This is the first study to demonstrate higher rates of picky eating in CCS than their peers, especially in CCS diagnosed at a young age. Published research into the general population has demonstrated the negative impact of a disrupted learning process for eating behaviour, which may then lead to the development of eating behaviours such as picky eating ${ }^{(2-4)}$. The knowledge that children often receive cancer treatment during this critical eating development phase disrupting the learning process, suggests that cancer therapy may have a lasting effect on a child's eating behaviour after treatment completion. In the general population children with picky eating consume less grains, fruits and vegetables than non-picky children resulting in low levels of vitamin E, vitamin C, folate and fibre, greatly impacting their diet quality ${ }^{(32,33)}$. Eating behaviours such as picky eating in CCS, are also likely to result in limited dietary intake and poor diet quality. Considering the importance of a healthy diet to reduce the risk of CVD and MetS $\mathrm{S}^{(34)}$, education focusing on eating behaviours needs to be considered.

Our study found several factors that may drive the diet quality of CCS. Diet quality, picky eating, overeating and BMI z-score were each represented in the three distinct clusters, suggesting they all may have a role in body size outcomes after treatment completion. In addition, comparison of cancer diagnostic classification with cluster group identification, indicated a distinct difference between the diagnosis of the child and the cluster they were allocated. This suggests that diagnosis groups which received single treatment modality regimens, such as Non-Hodgkin's lymphoma and Wilms' tumour, may be more likely to have a higher BMI z-score, altered eating behaviour and poor diet quality. These results may indicate an influence of treatment regimen on the four underlying variables that drive the cluster groups. Diagnosis groups such as brain tumour survivors that can receive more than one treatment modality (chemotherapy and radiation) might be more likely to have a lower BMI z-score, altered eating behaviour and poor diet quality. The findings are consistent with previous studies that have shown an increase in energy intake and BMI of Acute Lymphatic Leukaemia survivors who are considered to have a less intensive treatment regimen than other diagnostic categories ${ }^{(35)}$. The identification of this group through the use of cluster analysis provides a possible indication of which cancer diagnostic classification groups might be more likely to develop altered eating behaviour problems, poor diet quality and altered body size, allowing for targeted nutritional intervention after cancer treatment.

\section{Limitations}

This study was limited by a low response rate from CCS reducing the generalisability of results and allowing for potential bias within the small sample. However, low response rates are not unusual in the childhood cancer study population ${ }^{(36)}$. The small and heterogeneous nature of the sample may also have limited our ability to detect significant relationships between certain variables. Secondly, the reliance on parent-reports of their own and their child's height and weight resulted in a high proportion (22\%) of missing and possibly inaccurate data for this variable. In addition, the use of BMI z-scores to represent nutritional status is not ideal and prone to error in children with cancer, with BMI being a poor representation of body composition (37). Finally, the study design involved parent reported measures for diet and feeding variables. Despite being validated tools, the potential for reporter bias is high, especially for diet intake of the child and a parent's feeding behaviour with the child. This may have resulted in an over reporting of diet variety and underreporting of negative parent feeding behaviours. The use of more rigorous dietary intake measures such as a weighed food diary might be required for a more accurate measure of dietary intake in future studies.

\section{Conclusion}

This study found that CCS who have recently completed cancer treatment have a poor diet quality compared to age matched controls and have high rates of picky eating. Given that dietary habits established early in 
life are known to transpire into adulthood, and poor eating habits are linked to increased risk of Mets and CVD, young children who have recently completed cancer treatment with apparent poor dietary quality and altered eating behaviour are in critical need of dietary intervention early after cancer treatment. A tailored intervention that addresses altered eating behaviours, parent feeding practices and how to manage altered dietary intake due to treatment side effects is needed to bring about sustainable change for children early after cancer treatment.

\section{Acknowledgements}

The authors would like to thank Professor Richard Cohn and the Kids Cancer Centre, Sydney Children's Hospital, Randwick, NSW,

\section{Financial Support}

Dr Fleming was supported by a PhD scholarship from The Anthony Rothe Memorial Trust during the completion of this research (2011-2014). Claire Wakefield is supported by a Career Development Fellowship from the NHMRC of Australia (APP1143767). The Behavioural Sciences Unit is proudly supported by the Kids with Cancer Foundation. The BSU's survivorship research program is funded by a Cancer Council NSW Program Grant (PG16-02) with the support of the Estate of the Late Harry McPaul. These funding bodies did not have any role in the study, nor did they have a role in the writing of the manuscript or the decision to submit it for publication.

\section{Conflict of Interest}

No author has any commercial associations or conflicts of interest to disclose in relation to this manuscript

\section{Authorship}

Catharine A.K. Fleming - Lead investigator of study and contributed through formulating study question, designed study measures, conducted research, conducted data analysis and wrote manuscript.

Alexia J. Murphy - Aided data collection and provided extensive guidance through data analysis, interpretation of results and contributed to written manuscript.

Michael R. Fleming - Provided extensive statistical analysis guidance contributed to written manuscript

Jennifer Cohen - Aided in initial study conception through formulating study question and design and contributed to written manuscript.

Fiona Naumann - Aided data collection and provided extensive guidance through data analysis, interpretation of results and contributed to written manuscript.

Claire E Wakefield - Aided in initial study conception through formulating study questions and study design and contributed to written manuscript.

\section{References}

1. Australian Institute of Health and Welfare. (2012). A picture of Australia's children 2012. (Category No. PHE 167) Retrieved from http://www.aihw.gov.au/publication-detail/?id=10737423343

2. Birch LL, Fisher JO. Development of Eating Behaviors Among Children and Adolescents. Pediatrics. 1998;101(Supplement 2):539-49.

3. Sharp W, Jaquess D, Morton J, Herzinger C. Pediatric Feeding Disorders: A Quantitative Synthesis of Treatment Outcomes. Clinical Child and Family Psychology Review. 2010;13(4):348-65.

4. Manikam R, Perman JA. Pediatric Feeding Disorders. Journal of Clinical Gastroenterology. 2000;30(1):34-46.

5. Goddard E, Cohen J Bramley L, Wakefield CE, Beck EJ. Dietary intake and diet quality in children receiving treatment for cancer, Nutrition Reviews 2019: Volume 77, (5):267-277.

6. Andrassy RJ, \& Chwals WJ. Nutritional support of the pediatric oncology patient. Nutrition 1998;14: 124-129 
7. Bauer J, Jürgens H., \& Frühwald MC. Important Aspects of Nutrition in Children with Cancer. Advances in Nutrition: An International Review Journal 2011;2:67-77. doi: 10.3945/an.110.000141

8. Green R, Horn H, \& Erickson, JM. Eating Experiences of Children and Adolescents With Chemotherapy-Related Nausea and Mucositis. Journal of Pediatric Oncology Nursing 2010;27:209-216.

9. Sala, A., Pencharz, P., \& Barr, R. D. Children, cancer, and nutrition - A dynamic triangle in review. Cancer 2004;100:677-687. doi: 10.1002/cncr.11833

10. Fleming CA, Cohen, J, Murphy A, Wakefield CE, Cohn RJ, Naumann FL. Parent feeding interactions and practices during childhood cancer treatment. A qualitative investigation. Appetite. 2015;89:219-25.

11. Lesko LM. Psychosocial issues in the diagnosis and management of cancer cachexia and anorexia. Nutrition. 1989;5(2):114-6.

12. Hanigan MJ, Walter GA. Nutritional Support of the Child With Cancer. Journal of Pediatric Oncology Nursing. 1992;9(3):110-8.

13. Alvarez JA, Scully RE, Miller TL, Armstrong FD, Constine LS, Friedman DL, \& Lipshultz SE. Longterm effects of treatments for childhood cancers. Current Opinion in Pediatrics 2007;19:23-31.

14. Smith WA, Li C, Nottage KA, Mulrooney DA, Armstrong GT, Lanctot JQ, Chemaitilly W, Laver JH, Srivastava DK, Robison LL, Hudson MM. Lifestyle and metabolic syndrome in adult survivors of childhood cancer: a report from the St. Jude Lifetime Cohort Study. Cancer. 2014 Sep 1;120(17):274250 .

15. Cohen J, Wakefield CE, Fleming CA, Gawthorne R, Tapsell LC, Cohn RJ. Dietary intake after treatment in child cancer survivors. Pediatr Blood Cancer. 2012 May;58(5):752-7. doi: 10.1002/pbc.23280. Epub 2011 Aug 17. PMID: 21850679.Murphy-Alford, Alexia J, et al. "Body composition, dietary habits of theirintake and physical activity of young cancer-surviving children. Supportive Care in Cancer, 23 (2), 463-471survivors of childhood cancer." Clinical Nutrition 38.2 (2019): 842-847.

16. Zhang FF, Saltzman E, Kelly MJ, Liu S, Must A, Parsons SK, Roberts SB. Comparison of childhood cancer survivors' nutritional intake with US dietary guidelines. Pediatric blood \& cancer. 2015 Aug;62(8):1461-7.

17. Shams-White Marissa, et al. "Food craving and obesity in survivors of pediatric ALL and lymphoma." Appetite 96 (2016): 1-6.

18. Wardle J, Guthrie CA, Sanderson S, Rapoport L. Development of the Children's Eating Behaviour Questionnaire. The Journal of Child Psychology and Psychiatry and Allied Disciplines. 2001;42(07):96370.

19. Birch LL, Fisher JO, Grimm-Thomas K, Markey CN, Sawyer R, Johnson SL. Confirmatory factor analysis of the Child Feeding Questionnaire: a measure of parental attitudes, beliefs and practices about child feeding and obesity proneness. Appetite. 2001;36(3):201-10.

20. Watson J, Collins C, Sibbritt D, Dibley M, Garg M. Reproducibility and comparative validity of a food frequency questionnaire for Australian children and adolescents. International Journal of Behavioral Nutrition and Physical Activity. 2009;6(1):1-17.

21. Kuczmarski RJ, Ogden CL, \& Guo SS. Center for disease control and prevention growth charts for the United States: Methods and development. Health Statistics 2002;11(246):1-201.

22. Cole TJ, Flegal KM, Nicholls \& Jackson, AA. Body mass index cut offs to define thinness in children and adolescents: international survey. BMJ 2007;335 (7612):194.

23. Marshall S, Watson J, Burrows T, Guest M, Collins CE. The development and evaluation of the Australian child and adolescent recommended food score: a cross-sectional study. Nutrition Journal 2012;11(1):96.

24. NHMRC. Eat for Health, Educator Guide. In: Council NHaMR, editor. Canberra, Australia, 2013.

25. Dhonukshe-Rutten RAM, de Vries JHM, de Bree A, van der Put N, van Staveren WA, de Groot LCPGM. Dietary intake and status of folate and vitamin B12 and their association with homocysteine and cardiovascular disease in European populations. European Journal of Clinical Nutrition. 2009;63(1):18-30.

26. Bell L, Golley R, Daniels L, Magarey A. Dietary patterns of Australian children aged 14 and 24 months, and associations with socio-demographic factors and adiposity. European journal of clinical nutrition. 
2013;67(6):638-45.

27. Cameron A, Ball K, Pearson N, Lioret S, Crawford D, Campbell K, Hesketh K, McNaughton SA. Socioeconomic variation in diet and activity-related behaviours of Australian children and adolescents aged 2-16 years. Pediatric obesity. 2012;7(4):329-42.

28. Patrick H, Nicklas TA. A review of family and social determinants of children's eating patterns and diet quality. Journal of the American College of Nutrition. 2005;24(2):83-92.

29. Ventham JC, Reilly JJ. Childhood leukaemia: a model of pre-obesity. Proceedings of the Nutrition Society. 1999;58(2):277-81.

30. Commonwealth Scientific Industrial Research Organisation. Australian National Children's Nutrition and Physical Activity Survey- Main Findings. In: Department of Health and Ageing, editor.: Australian Government; 2007.

31. Galloway AT, Fiorito L, Lee Y, Birch LL. Parental pressure, dietary patterns, and weight status among girls who are "picky eaters". Journal of the American Dietetic Association. 2005;105(4):541-8.

32. Jacobi C, Schmitz G, Agras WS. Is picky eating an eating disorder? International Journal of Eating Disorders. 2008;41(7):626-34.

33. NHMRC. Australian Dietary Guidelines. In: Council NHaMR, editor. Canberra 2013.

34. Hansen JA, Stancel HH, Klesges LM, Tyc VL, Hinds PS, Wu S, Hudson MM, Kahalley LSEating Behavior and BMI in Adolescent Survivors of Brain Tumor and Acute Lymphoblastic Leukemia. Journal of Pediatric Oncology Nursing. 2014;31(1):41-50.

35. Wakefield CE, Fardell JE, Doolan EL, Aaronson NK, Jacobsen PB, Cohn RJ, King M. Participation in psychosocial oncology and quality-of-life research: a systematic review. The Lancet Oncology. 2017 1;18(3):e153-65.

36. Murphy AJ, White M, Davies PSW. Body composition of children with cancer. The American Journal of Clinical Nutrition 2010;92:55-60. doi: 10.3945/ajcn.2010.29201

37. Cole TJ, Flegal, KM, Nicholls D Jackson, AA Body mass index cut offs to define thinness in children and adolescents: international survey. Bmj 2007, 335 (7612), 194.

\section{Hosted file}

TABLE 1 Demographic characteristics of parents and children PBC.pdf available at https: //authorea.com/users/390450/articles/504792-poor-diet-quality-and-adverse-eatingbehaviours-in-young-survivors-of-childhood-cancer

\section{Hosted file}

TABLE 2 Clinical characteristics of children who had recently completed cancer treatment PBC.pdf available at https://authorea.com/users/390450/articles/504792-poor-diet-quality-andadverse-eating-behaviours-in-young-survivors-of-childhood-cancer

\section{Hosted file}

TABLE 3 Proportion (\%) of energy from core and non-core foods for participants PBC.pdf available at https://authorea.com/users/390450/articles/504792-poor-diet-quality-andadverse-eating-behaviours-in-young-survivors-of-childhood-cancer

\section{Hosted file}

TABLE 4 The Australian Child and Adolescent Recommended Food Score outcomes PBC.pdf available at https://authorea.com/users/390450/articles/504792-poor-diet-quality-and-adverseeating-behaviours-in-young-survivors-of-childhood-cancer

\section{Hosted file}

TABLE 5 Parent reported child eating behaviour outcomes pbc.pdf available at https: //authorea.com/users/390450/articles/504792-poor-diet-quality-and-adverse-eatingbehaviours-in-young-survivors-of-childhood-cancer 


\section{Hosted file}

TABLE 6 Descriptive statistics of the characteristics for each cluster group PBC.pdf available at https://authorea.com/users/390450/articles/504792-poor-diet-quality-andadverse-eating-behaviours-in-young-survivors-of-childhood-cancer 